\title{
Effects of vitamins $A$ and $E$ supplementation on the pharmacokinetics and toxicity profile of aspirin in rats
}

\author{
Mohammed A. Mahdi', Abdulrahman U. Zezi ${ }^{1}$, Ibrahim A. Aguye', Janet I. Ejiofor ${ }^{1}$, Musa A. Usman² \\ ${ }^{I}$ Pharmacology and Therapeutics, Faculty of Pharmaceutical Sciences, Ahmadu Bello University, Zaria, Nigeria, \\ ${ }^{2}$ Pharmaceutical and Medicinal Chemistry, Ahmadu Bello University, Zaria, Nigeria
}

The main aim of this study is to observe improveement in the utilization of aspirin through the reduction of untoward effects by studying the pharmacokinetic profile of a non-compartmental model and toxicity with or without vitamins A, E or combinations following oral administration in rats. The Trinder (1954) method was adopted to estimate the serum concentrations of salicylic acid after prior validation. The pharmacokinetic parameters were generated by the method of residuals in four (4) groups of aspirin alone, aspirin plus vitamin $\mathrm{A}$, aspirin plus vitamin $\mathrm{E}$ and aspirin plus combinations of vitamins A and E groups respectively. Data obtained were presented as Mean +/- SEM and analyzed by one way ANOVA. Coadministration of $10 \mathrm{mg} / \mathrm{kg}$ with vitamins $\mathrm{A}$ had no significant effect on all pharmacokinetic parameters. Contrarily, coadministration of aspirin with vitamin $\mathrm{E}$ or combinations of vitamins $\mathrm{A}$ and $\mathrm{E}$ resulted in the following changes in aspirin pharmacokinetics; AUC 0-6 significantly decreased to $88.1+/-13.19$ in aspirin plus vitamin A ana E group compared to aspirin plus vitamin A group $(105.24+/-6.71 \mathrm{ugh} / \mathrm{mL}$, AUC 6-infinity significantly increased to 38.8 +/- $13.07 \mathrm{ugh} / \mathrm{mL}$ in ASA plus vitamin E group compared to all the three (3) groups, Kel decreased significantly to 0.74 +/- 0.15 h-1 in aspirin plus vitamin E group compared to all the three groups, the elimination half-life t 1/2 increased significantly in aspirin plus vitamin E group compared to all the three (3) groups, total clearance (CLT) significantly increased to $118.43+/-13.53$ in aspirin plus vitamin A and E group compared to aspirin plus vitamin A and aspirin plus vitamin E group respectively.

The histopathology results recorded indicated that supplementation with vitamin $\mathrm{E}$ or combinations of vitamins $\mathrm{A}$ and $\mathrm{E}$ significantly decreased liver and kidney toxicity caused by 10,50 and to some extent $100 \mathrm{mg} / \mathrm{kg}$ aspirin. Vitamin A supplementation has shown a protective effect on the myocardium at the three (3) dose levels observed. 